\title{
Spontaneous electro-weak symmetry breaking and cold dark matter
}

\author{
Shou-hua Zhu \\ Institute of Theoretical Physics, School of Physics, Peking University, Beijing 100871, China
}

(Dated: April 16, 2021)

\begin{abstract}
In the standard model, the weak gauge bosons and fermions obtain mass after spontaneous electroweak symmetry breaking, which is realized through one fundamental scalar field, namely Higgs field. In this paper we study the simplest scalar cold dark matter model in which the scalar cold dark matter also obtains mass through interaction with the weak-doublet Higgs field, the same way as those of weak gauge bosons and fermions. Our study shows that the correct cold dark matter relic abundance within $3 \sigma$ uncertainty $\left(0.093<\Omega_{d m} h^{2}<0.129\right)$ and experimentally allowed Higgs boson mass $\left(114.4 \leq m_{h} \leq 208 \mathrm{GeV}\right)$ constrain the scalar dark matter mass within $48 \leq m_{S} \leq 78 \mathrm{GeV}$. This result is in excellent agreement with that of W. de Boer et.al. $(50 \sim 100 \mathrm{GeV})$. Such kind of dark matter annihilation can account for the observed gamma rays excess $(10 \sigma)$ at EGRET for energies above $1 \mathrm{GeV}$ in comparison with the expectations from conventional Galactic models. We also investigate other phenomenological consequences of this model. For example, the Higgs boson decays dominantly into scalar cold dark matter if its mass lies within $48 \sim 64 \mathrm{GeV}$.
\end{abstract}

PACS numbers: 14.80.-j, 14.60.Fr, 11.15.Ex

Understanding the mechanism of electro-weak symmetry breaking (EWSB) is a primary goal of the Large Hadron Collider (LHC) and International Linear Collider (ILC). In the standard model (SM), EWSB is realized through one fundamental Higgs field, namely the vacuum expectation value (VEV) of Higgs field $\langle\Phi\rangle \neq 0$. After EWSB the weak gauge bosons and fermions obtain mass, and only one neutral Higgs boson is left in particle spectrum. The couplings among Higgs boson and weak gauge bosons/fermions are proportional to their corresponding mass. This is one of the most important features of spontaneous EWSB. Though this feature has not been directly tested in the past experiments, the SM predictions and experimental measurements are in good agrement at an accuracy of $O(0.1 \%)$. The global fit of LEP, SLD and Tevatron data predicts the Higgs boson mass to be $m_{h}=98_{-36}^{+52} \mathrm{GeV}$ and $m_{h}<208 \mathrm{GeV}$ at $95 \%$ CL using latest preliminary top quark mass $m_{t}=174.3 \pm 3.4$ $\mathrm{GeV}$ [1]. The latest direct search sets the lower bound of SM Higgs boson of $114.4 \mathrm{GeV}$ at $95 \%$ confidence level (CL) 2]. Because EWSB is the only untouched part in the SM, it is most likely that the new physics is closely related to the Higgs sector.

Over the past several years our understanding of components of the Universe has undergone important advances. The existence of dark matter (DM) is by now well established. The non-baryonic cold dark matter density is (within $3 \sigma$ uncertainty) [3]

$$
0.093<\Omega_{d m} h^{2}<0.129
$$

where $h \approx 0.71$ is the normalized Hubble expansion rate. However the microscopic properties of dark matter are remarkably unconstrained. Therefore it is quite interesting to ask how cold dark matter interacts with usual SM matter. Though the cold dark matter is allowed to feel weak interaction, such possibility will usually involve more free parameters. The popular cold dark matter candidate neutralino in supersymmetrical models is a good example. Therefore in this paper we only discuss the case that dark matter is SM gauge group singlet.

Motivated by the successful assumption that weak gauge bosons and fermions obtain mass from the interactions with weak-doublet Higgs field, it is quite natural to assume that SM gauge group singlet cold dark matter obtains mass the same way as those of weak gauge bosons and fermions. Thus the coupling among Higgs boson and cold dark matter is solely fixed by dark matter mass. If the dark matter is fermion, it will obtain mass through dimension-five non-renormalizeble operator $(\bar{\Psi} \Psi)\left(\Phi^{+} \Phi\right)$ with $\Phi$ the usual weak-doublet Higgs field. Thus we prefer to take the cold dark matter as scalar field, denoted as S. In fact the scalar particle as cold dark matter has been widely studied in literature [4, 5]. If we introduce only one singlet scalar field $\mathrm{S}$, there is only one extra free parameter other than those in the SM, namely the mass of the scalar cold dark matter $m_{S}$. In this paper we will study this simplest cold scalar dark matter model. Our results show that the correct cold dark matter relic density in Eq. (11) and experimentally allowed Higgs boson mass $\left(114.4 \leq m_{h} \leq 208 \mathrm{GeV}\right.$ [13] ) constrain the scalar dark matter within $48 \leq m_{S} \leq 78 \mathrm{GeV}$. This result is in excellent agreement with that of $\mathrm{W}$. de Boer et.al. $(50 \sim 100 \mathrm{GeV})[6]$. Such kind of dark matter annihilation can account for the observed gamma rays excess $(10 \sigma)$ at EGRET for energies above $1 \mathrm{GeV}$ in comparison with the expectations from conventional Galactic models. In this paper we also investigate other phenomenological consequences of this model. For example, the Higgs boson decays dominantly into scalar cold dark matter if its mass lies within $48 \sim 64 \mathrm{GeV}$.

The Lagrangian of the simplest model can be written as

$$
L=L_{S M}+\frac{1}{2} \partial_{\mu} S \partial^{\mu} S-\frac{\lambda_{S}}{4} S^{4}-\lambda S^{2}\left(\Phi^{+} \Phi\right)
$$

where $L_{S M}$ is the Lagrangian of the SM and $\Phi$ is the weak doublet Higgs field. $L$ is obviously invariant un- 
der discrete transformation $S \rightarrow-S$, which ensures $\mathrm{S}$ the good candidate of cold dark matter. The model discussed in this paper is different from that of Ref. [5]. The main difference is that the mass term of S field $m_{0}^{2} S^{2}$ is not included in this paper. It is known that the mass of dark matter can vary many orders of magnitude depending on the strength it interacts with usual matter. Here we investigate the possibility that dark matter is in the weak scale while $m_{0}^{2}$ can be anything. Therefore it is unnecessary to introduce one extra new mass scale. In this paper we assume that weak doublet Higgs field solely induces mass for all particles: weak gauge bosons, fermions and singlet scalar field $S$. The model can be a massless one before EWSB. The spontaneous symmetry breaking, trigger by $\mu^{2} \Phi^{+} \Phi$, can be induced radiatively [7]. On the contrary $S^{2}$ term is absent or negligibly small provided that it does not get contribution from $\lambda S^{2}\left(\Phi^{+} \Phi\right)$. After spontaneous electro-weak symmetry breaking $\langle\Phi\rangle=v=246 \mathrm{GeV}$, the Higgs boson, as in the standard model, $m_{h}^{2}=\lambda_{h} v^{2}$ with $\lambda_{h}$ the coefficient of $\left(\Phi^{+} \Phi\right)^{2}$ and $m_{S}^{2}=\lambda v^{2}$. It is obvious that coupling $\lambda$ is determined by $m_{S}$ and in this model $\lambda$ is the only extra free parameter relevant to our discussion.

Next we will explore the cosmological constraints on this model by demanding the present abundance of $\mathrm{S}$ to be in the range of Eq. (11). As we can see, this imposes a very strong relationship between Higgs boson mass and $m_{S}$. The calculation of S abundance follows the standard procedure [8] and we refer the interesting reader to Ref. [5] for the details.

The present density of $\mathrm{S}$ can be written as [5]

$$
\Omega_{S} h^{2}=\frac{\left(1.07 \times 10^{9}\right) x_{f}}{\sqrt{g_{*}} M_{p l}[\text { in } \mathrm{GeV}]<\sigma v_{r e l}>},
$$

where $g_{*}$ counts the degrees of freedom in equilibrium at annihilation, $x_{f}$ is the inverse freeze-out temperature in units of $m_{S}$, which can be obtained by solving the equation

$$
x_{f} \simeq \ln \left[\frac{0.038 M_{p l} m_{S}<\sigma v_{r e l}>}{\sqrt{g_{*} x_{f}}}\right] .
$$

In Eqs. (3) and (4), $v_{\text {rel }}$ is the relative velocity of the two incoming dark matter particles, $M_{p l}$ is the Planck mass and $\langle\ldots\rangle$ denotes the relevant thermal average.

Since the scalar dark matter obtains mass through interaction with VEV of the Higgs field, it is natural to expect that dark matter mass scale is $O(100) \mathrm{GeV}$. Therefore $\sigma v_{r e l}$ can be obtained by evaluating the tree-level diagram in Fig. 1. In the non-relativistic limit, $\sigma v_{r e l}$ is [5]

$$
\begin{aligned}
\sigma_{a n n} v_{r e l} & =\frac{8 \lambda^{2} v^{2}}{\left(4 m_{S}^{2}-m_{h}^{2}\right)^{2}+m_{h}^{2} \Gamma_{h}^{2}} F_{X} \\
& =\frac{8 m_{S}^{4}}{v^{2}\left[\left(4 m_{S}^{2}-m_{h}^{2}\right)^{2}+m_{h}^{2} \Gamma_{h}^{2}\right]} F_{X}
\end{aligned}
$$

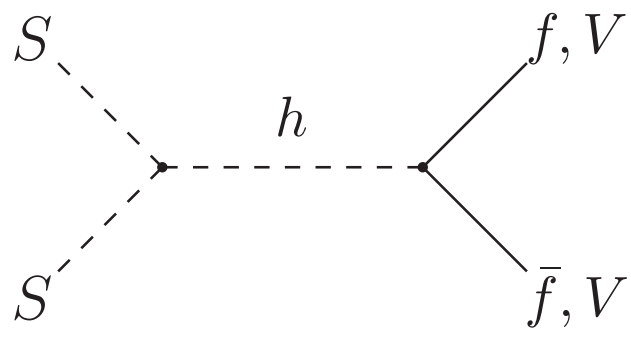

FIG. 1: Schematic Feynman diagram for $S S \rightarrow$ SM particles. Here $\mathrm{f}$ and $\mathrm{V}$ represent SM fermions and weak gauge bosons respectively.

with

$$
F_{X}=\lim _{m_{\tilde{h}} \rightarrow 2 m_{S}}\left(\frac{\Gamma_{\tilde{h} \rightarrow X}}{m_{\tilde{h}}}\right) .
$$

Here $\Gamma_{h}$ is the Higgs total decay width and $\Gamma_{\tilde{h} \rightarrow X}$ denotes the partial decay width for the virtual $\tilde{h}$ decay into $X$, $\tilde{h} \rightarrow X$, in the limit $m_{\tilde{h}} \rightarrow 2 m_{S}$. Here X represents SM particles.

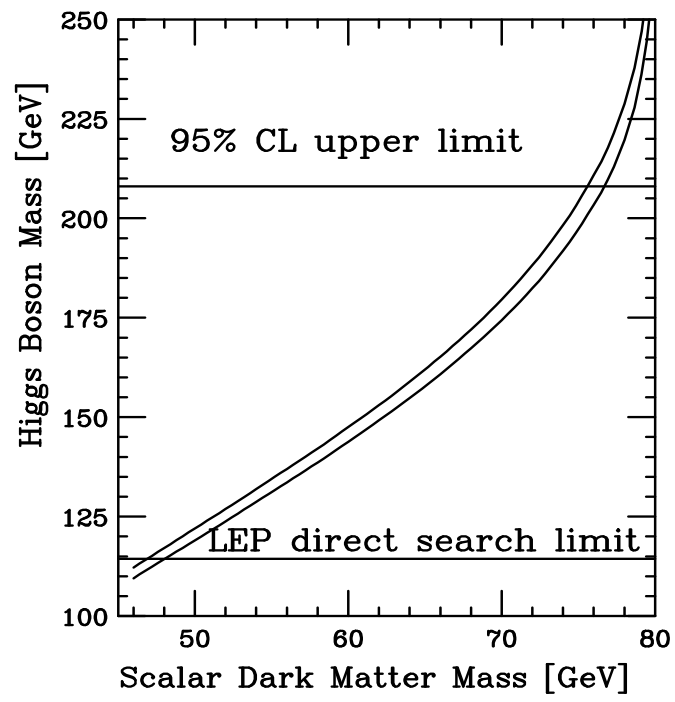

FIG. 2: Allowed Higgs boson and scalar dark matter mass region (between two curves). This mass region can produce correct relic density $0.093<\Omega_{d m} h^{2}<0.129$ (within $3 \sigma$ uncertainty). Also shown are Higgs boson upper $(208 \mathrm{GeV})$ and lower $(114.4 \mathrm{GeV})$ mass limits from electro-weak precision data global fit and direct search at LEP.

In Fig. 2 and table 1 we show the allowed Higgs boson and scalar dark matter mass region [between two curves], provided that relic abundance satisfies Eq. (1). From figure we can see a very strong relationship between Higgs boson mass and $m_{S}$. It is known that the 
coupling strength among Higgs boson and SM particles is proportional to their corresponding mass, as we discussed above. At the same time, the coupling between Higgs boson and scalar dark matter is also proportional to $m_{S}$. Once $m_{S}$ is fixed, the only free varying parameter is $m_{h}$. If we impose the Higgs mass constraints from direct search limit $114.4 \mathrm{GeV}$ to $208 \mathrm{GeV}$, the allowed scalar dark matter is

$$
48 \leq m_{S} \leq 78 \mathrm{GeV} .
$$

It should be noted that $m_{S}$ can't be larger than $m_{W}$. Otherwise the cross section of $S S \rightarrow W W$ becomes too large and the relic abundance is out of the region in Eq. (11). Eq. (77) is in excellent agreement with that of W. de Boer et.al. $(50 \sim 100 \mathrm{GeV})[6]$. Such kind of dark matter annihilation produces mono-energetic quarks of $50 \sim 100$ $\mathrm{GeV}$, which can account for the observed gamma rays excess $(10 \sigma)$ at EGRET for energies above $1 \mathrm{GeV}$ in comparison with the expectations from conventional Galactic models. It should be emphasized that the crucial part of EGRET photon excess origin is mono-energetic quarks, no matter which kind of dark matter annihilation produces them. In fact the further investigation by W. de Boer et.al. 9] shows that supersymmetric models with suitable parameter space are compatible with their conjecture. There the dark matter candidate is neutralino.

It is also interesting to note that for Eq. (7), $F_{V V^{*}}$ with $V^{*}$ the virtual $W$ and $Z$ plays a very important role. $F_{V V^{*}}$ can be extracted from Ref. [10] as

$$
F_{V V^{*}}=\frac{3 G_{F}^{2} m_{V}^{4}}{16 \pi^{3}} \delta_{V} R(x)
$$

with $\delta_{W}=1, \delta_{Z}=7 / 12-10 / 9 \sin ^{2} \theta_{W}+40 / 9 \sin ^{4} \theta_{W}$ $\left(\theta_{W}\right.$ is the weak angle). Here

$$
\begin{aligned}
R(x)= & \frac{3\left(1-8 x+20 x^{2}\right)}{\sqrt{4 x-1}} \arccos \left(\frac{3 x-1}{2 x^{3 / 2}}\right) \\
& -\frac{1-x}{2 x}\left(2-13 x+47 x^{2}\right) \\
& -\frac{3}{2}\left(1-6 x+4 x^{2}\right) \log x
\end{aligned}
$$

with $x=m_{V}^{2} /\left(4 m_{S}^{2}\right)$.

\begin{tabular}{l|cc}
\hline \hline$m_{S}[\mathrm{GeV}]$ & $m_{h}$ upper limit $[\mathrm{GeV}]$ & $m_{h}$ lower limit $[\mathrm{GeV}]$ \\
\hline 50 & 122 & 119 \\
55 & 134 & 131 \\
60 & 148 & 144 \\
65 & 162 & 158 \\
70 & 180 & 174 \\
75 & 204 & 197 \\
80 & 275 & 261 \\
\hline \hline
\end{tabular}

TABLE I: Upper and lower limits on Higgs boson for several $m_{S}$ in order to obtain the correct relic abundance in Eq. (1).
Fig. 2 and table $\llbracket$ also show that in the allowed mass region, $m_{h}>2 m_{S}$. This feature has very important phenomenological consequence, namely the Higgs can decay into pair of dark matter. The partial decay width can be written as

$$
\Gamma(h \rightarrow S S)=\frac{m_{S}^{4}}{8 \pi v^{2} m_{h}} \sqrt{1-\frac{4 m_{S}^{2}}{m_{h}^{2}}} .
$$

$\Gamma(h \rightarrow S S)$ and $\operatorname{Br}(h \rightarrow S S)$ are shown in Fig. 3 and 4. From Fig. 3 we can see that the partial decay width is between $0.02 \sim 0.08 \mathrm{GeV}$ for $m_{S}=48 \sim 78 \mathrm{GeV}$. In Fig. 4 the rapid drop around $m_{S}=65 \mathrm{GeV}$ is due to the rapid growth of $\Gamma\left(H \rightarrow V V^{*}\right)$. From Fig. 四 we can see good and bad news. The good news is that there is certain possibility that Higgs boson can decay into SM particle, especially for $m_{S} \geq 64 \mathrm{GeV}$. Therefore we still have opportunity to see Higgs boson resonance as the case in the SM. The bad news is that we need more luminosity for the usual Higgs search strategies, especially for $m_{S} \leq$ $60 \mathrm{GeV}$. Further efforts should be put on search of the invisibly Higgs boson decay [11].

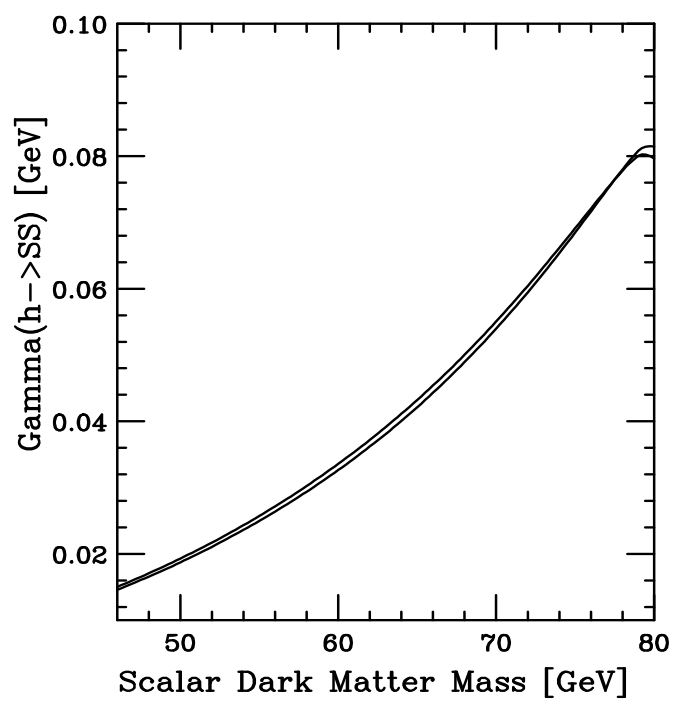

FIG. 3: Decay width [in GeV] of $h \rightarrow S S$ as a function of $m_{S}$. Region between two curves can produce correct cold dark matter relic abundance in Eq.(1).

In this paper we study the simplest scalar cold dark matter model in which the scalar cold dark matter obtains mass through interaction with the weak-doublet Higgs field, the same way as those of weak gauge bosons and fermions. The coupling between Higgs boson and scalar dark matter is solely fixed by scalar dark matter mass. The correct cold dark matter relic abundance within $3 \sigma$ uncertainty $\left(0.093<\Omega_{d m} h^{2}<0.129\right)$ and experimentally allowed Higgs boson mass $\left(114.4 \leq m_{h} \leq\right.$ $208 \mathrm{GeV}$ ) constrain the scalar dark matter mass within $48 \leq m_{S} \leq 78 \mathrm{GeV}$. This result is in excellent agreement with that of W. de Boer et.al. $(50 \sim 100 \mathrm{GeV})$. Such 


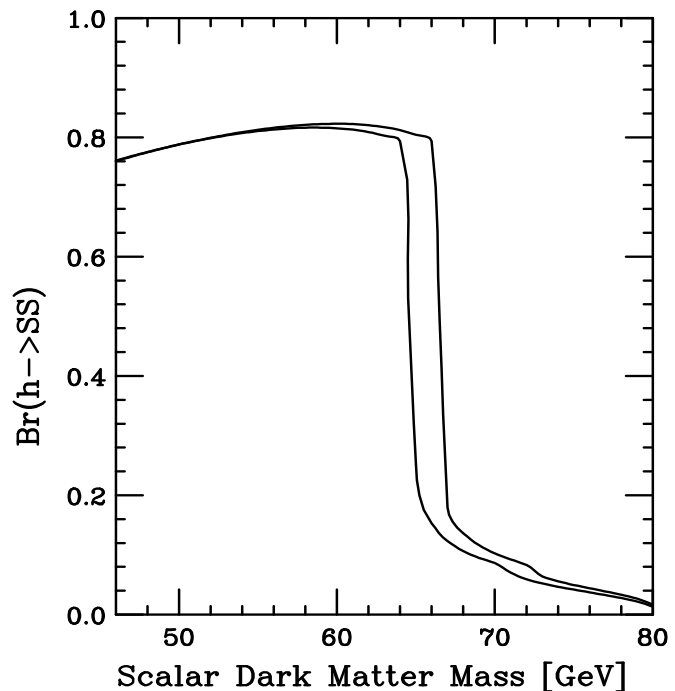

FIG. 4: Branching ratio of $h \rightarrow S S$ as a function of $m_{S}$. Other conventions are the same with Fig. 3 .

kind of dark matter annihilation can account for the ob- served gamma rays excess $(10 \sigma)$ at EGRET for energies above $1 \mathrm{GeV}$ in comparison with the expectations from conventional Galactic models. We also investigate other phenomenological consequences of this model. Most importantly the Higgs boson decays dominantly into scalar cold dark matter if its mass lies within $48 \sim 64 \mathrm{GeV}$.

Recently Bergstrom et. al. [12] investigated the internal consistency of the halo dark matter model which has been proposed by de Boer et. al. Certain inconsistency is found 12]. Now that the proposal of de Boer et. al. is still in debate, we should emphasize that our main results, i.e. (1) the correlation between masses of scalar dark matter and Higgs boson, (2) the Higgs boson can decay into dark matter with large branching ratio, might be irrelevant to the EGRET data. However LHC/ILC can provide ideal place to investigate the scalar dark matter in the Higgs boson decay [11].

Acknowledgements: The author thanks Prof. Y.Q. Ma for the discussion on EGRET, and Prof. C. Liu and H.Q. Zheng for the discussion on Eq. (2). This work was supported in part by the Natural Sciences Foundation of China under grant No. 90403004, the trans-century fund and the key grant project (under No. 305001) of Chinese Ministry of Education.
[1] A. Juste, arXiv:hep-ex/0511025

[2] R. Barate et al. [LEP Working Group for Higgs boson searches], Phys. Lett. B 565, 61 (2003).

[3] S. Eidelman et al. [Particle Data Group], Phys. Lett. B 592 (2004) 1.

[4] M. J. G. Veltman and F. J. Yndurain, Nucl. Phys. B 325, 1 (1989); V. Silveira and A. Zee, Phys. Lett. B 161, 136 (1985); J. McDonald, Phys. Rev. D 50, 3637 (1994). H. Davoudiasl, R. Kitano, T. Li and H. Murayama, Phys. Lett. B 609, 117 (2005).

[5] C. P. Burgess, M. Pospelov and T. ter Veldhuis, Nucl. Phys. B 619, 709 (2001) arXiv:hep-ph/0011335.

[6] W. de Boer, C. Sander, A. V. Gladyshev and D. I. Kazakov, arXiv:astro-ph/0508617.

[7] S. R. Coleman and E. Weinberg, Phys. Rev. D 7, 1888 (1973).

[8] E.W. Kolb and M.S. Turner, The Early Universe, Addison-Wesley, 1990.

[9] W. de Boer, C. Sander, V. Zhukov, A. V. Gladyshev and
D. I. Kazakov, arXiv:hep-ph/0511154

[10] A. Djouadi, arXiv:hep-ph/0503172 and references therein.

[11] S. h. Zhu, Eur. Phys. J. C 47, 833 (2006) arXiv:hep-ph/0512055 and references therein.

[12] L. Bergstrom, J. Edsjo, M. Gustafsson and P. Salati, JCAP 0605, 006 (2006) arXiv:astro-ph/0602632; for the earilier invesigations to see, for exmple, A. Cesarini, F. Fucito, A. Lionetto, A. Morselli and P. Ullio, Astropart. Phys. 21, 267 (2004) arXiv:astro-ph/0305075; L. Bergstrom, P. Ullio and J. H. Buckley, Astropart. Phys. 9, 137 (1998) arXiv:astro-ph/9712318; A. Bottino, F. Donato, N. Fornengo and S. Scopel, Phys. Rev. D 70, 015005 (2004) arXiv:hep-ph/0401186.

[13] Because the singlet scalar only interacts with Higgs boson, current electroweak precision data is not sensitive to such kind of new interactions beyond the SM. Therefore we assume that experimentally allowed Higgs boson mass in the SM is not affected. 